\title{
Activation of pannexin-1 mediates triglyceride-induced macrophage cell death
}

\author{
Byung Chul Jung ${ }^{1,2}$, Sung Hoon Kim ${ }^{2,3}$, Jaewon Lim ${ }^{2,4, *} \mathcal{E}$ Yoon Suk Kim ${ }^{2, *}$ \\ ${ }^{1}$ Department of Nutritional Sciences and Toxicology, University of California, Berkeley, CA 94720, United States, ${ }^{2}$ Department of \\ Biomedical Laboratory Science, College of Health Sciences, Yonsei University, Wonju 26493, ${ }^{3}$ Department of Biomedical Laboratory \\ Science, Korea Nazarene University, Cheonan 31172, ${ }^{4}$ Department of Biomedical Laboratory Science, College of Medical Sciences, \\ Daegu Haany University, Gyeongsan 38610, Korea
}

The accumulation of triglycerides (TGs) in macrophages induces cell death, a risk factor in the pathogenesis of atherosclerosis. We had previously reported that TG-induced macrophage death is triggered by caspase- 1 and -2 , therefore we investigated the mechanism underlying this phenomenon. We found that potassium efflux is increased in TG-treated THP-1 macrophages and that the inhibition of potassium efflux blocks TG-induced cell death as well as caspase-1 and -2 activation. Furthermore, reducing ATP concentration (known to induce potassium efflux), restored cell viability and caspase-1 and -2 activity. The activation of pannexin-1 (a channel that releases ATP), was increased after TG treatment in THP-1 macrophages. Inhibition of pannexin-1 activity using its inhibitor, probenecid, recovered cell viability and blocked the activation of caspase-1 and -2 in TG-treated macrophages. These results suggest that TG-induced THP-1 macrophage cell death is induced via pannexin-1 activation, which increases extracellular ATP, leading to an increase in potassium efflux. [BMB Reports 2020; 53(11): 588-593]

\section{INTRODUCTION}

Atherosclerosis is a disease characterized by the narrowing and hardening of arteries due to the formation of lipid-laden plaques, often leading to ischemic cardiomyopathy and stroke (1). Since the abnormal accumulation of lipoproteins, especially oxidative low-density lipoproteins (Oxi-LDL), is considered the

*Corresponding authors. Yoon Suk Kim, Tel: +82-33-760-2860; Fax: +82-33-760-2195; E-mail: yoonsukkim@yonsei.ac.kr; Jaewon Lim, Tel: +82-53-819-1352; Fax: +82-53-819-1353; E-mail: jaewon330@dhu. ac.kr

https://doi.org/10.5483/BMBRep.2020.53.11.179

Received 27 August 2020, Revised 10 September 2020, Accepted 28 September 2020

Keywords: Cell death, Pannexin-1, Potassium efflux, THP-1 macrophages, Triglyceride (TG) first step in the pathogenesis of atherosclerosis, many studies have been directed towards understanding the characteristics of Oxi-LDL and oxidative stress $(2,3)$. Multiple lines of evidence support that elevated TG levels are associated with an increased risk of atherosclerosis and coronary heart disease $(4,5)$. We have previously demonstrated that TGs induced the macrophage death via caspase- 1 and $2(6,7)$. These studies suggested a putative mechanism by which TG contributes to the development of atherosclerosis. However, the mechanism by which TG triggers the caspase cascade in macrophages remains elusive.

Potassium efflux occurs during cell death due to loss of ionic homeostasis. Furthermore, dysregulation of potassium efflux is one of the causative factors of caspase-dependent cell death (8). For example, the potassium ionophore valinomycin can induce potassium efflux, which triggers caspase- 3 activation and subsequent cell death in $\mathrm{CHO}$ cells (9). Furthermore, $\alpha$-toxinmediated caspase-2 activation and cell death were suppressed on preventing potassium efflux in HeLa cells, indicating that potassium efflux is a prerequisite for caspase-2-mediated cell death (10). Many studies have speculated on the mechanisms by which potassium efflux is regulated. One of the well-known mechanisms for increasing potassium efflux is the opening of the ATP-sensitive potassium channel (11). It has also been reported that ATP-sensitive potassium channels open during the initial phase of myocardial ischemia, one of the consequences of atherosclerosis (12).

Pannexins are large pore-forming channels and consist of three family members (pannexin-1, 2, and 3). Since pannexin-1 is ubiquitously expressed ATP-permeable channel and is involved in a variety of cellular processes, including cell death, it has attracted significant attention in research (13). In the current study, we investigated the involvement of potassium efflux in TG-induced macrophage cell death. We report that TG induces an increase of extracellular ATP via pannexin-1, which in turn activates ATP-sensitive potassium channels, resulting in an increase in potassium efflux and subsequent activation of the caspase cascade in THP-1 macrophages. These results suggest that TG-induced cell death of macrophages is mediated by the activation of pannexin-1. 


\section{RESULTS}

\section{Potassium efflux is involved in TG-induced THP-1 macrophage cell death}

Though previous studies have reported that TG-induced cell death is mediated by caspase- 1 and $2(6,7)$, the mechanism by which they are activated is not fully understood. Several studies have reported that the release of intracellular potassium ions activates caspases $(14,15)$. Therefore, this study investigated whether potassium efflux is involved in TG-induced caspasemediated THP-1 macrophage death. When extracellular potassium concentration was measured in PMA-differentiated THP-1 macrophages (incubated with or without $1 \mathrm{mg} / \mathrm{ml}$ of TG for $24 \mathrm{~h}$ ), it was found to be higher in TG-treated macrophages (Fig. 1A). Furthermore, when macrophages were treated with glyburide, an inhibitor of ATP-sensitive potassium channels, TG-induced cell death was restored in a dose-dependent manner (Fig. 1B). When the extracellular concentration of potassium was increased via treatment with $25 \mathrm{mM} \mathrm{KCl}$ to prevent the release of potas- sium ions, cell viability was recovered (Fig. 1C). Suppression of potassium efflux by treating macrophages with glyburide reduced the levels of cleaved PARP and several caspases (caspase-3, 7, 8, and 9) that are known to be responsible for TG-induced macrophage death (Fig. 1D). When THP-1 macrophages were incubated with TG in the presence of glyburide, treatment with glyburide decreased the activity of caspase- 1 which is reported to induce the activity of caspase-3, -7, -8, and -9 (7) (Fig. 1E and 1F). Treatment with glyburide also reduced the activity of caspase-2, a known upstream molecule of caspase-1 (7), which increased upon TG treatment (Fig. 1G). These results demonstrate that potassium efflux induces TGstimulated macrophage death via the activation of caspases.

\section{The increase in extracellular ATP leads to TG-triggered macrophage cell death}

It has recently been reported that an increase in extracellular ATP affects ATP-sensitive potassium channels, thereby increasing potassium efflux (16). Therefore, to determine if extracellular
A

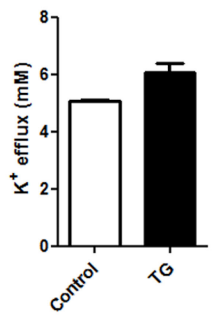

D

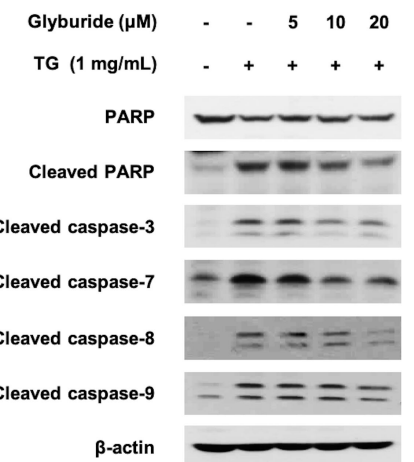

B

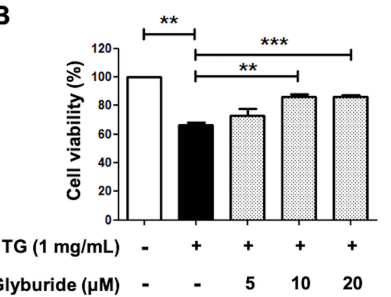

E

Glyburide $(\mu \mathrm{M}) \quad-\quad-5 \quad 5 \quad 10 \quad 20$

TG $(1 \mathrm{mg} / \mathrm{mL})+++++$

Caspase-1

Cleaved

caspase-1

$\beta$-actin

F

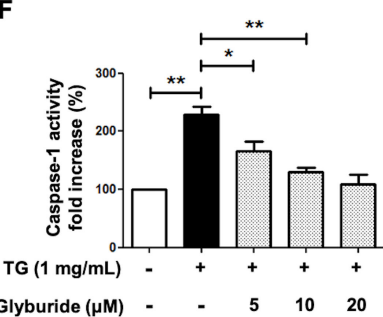

C

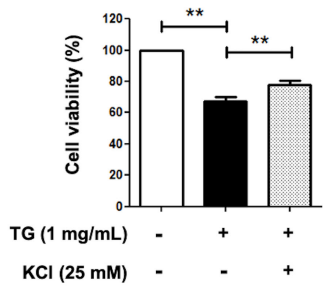

G

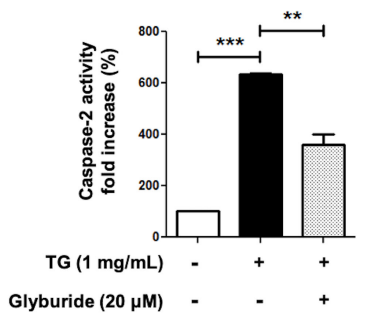

Fig. 1. Potassium efflux is involved in TG-induced THP-1 macrophage cell death. (A) THP-1 cells were differentiated with PMA and incubated with or without TG for an additional $24 \mathrm{~h}$. Extracellular potassium concentration was measured by atomic absorption spectrometry. (B) THP-1 macrophages were incubated with TG in the absence or presence of the potassium efflux inhibitor, glyburide. Viable cells were enumerated by the trypan blue dye exclusion assay. The number of viable cells in THP-1 macrophages without TG treatment was set as $100 \%$. (C) THP-1 macrophages were incubated with TG in the presence of $\mathrm{KCl}$ for $24 \mathrm{~h}$ and the trypan blue exclusion assay was performed. (D) The cleaved form of PARP, and the cleavage of caspase-3, -7, -8 and -9 , were detected by Western blotting. (E) The cleavage of caspase-1 was detected by Western blotting. (F) THP-1 macrophages were incubated with TG in the presence of glyburide for $24 \mathrm{~h}$, after which caspase-1 activity was assessed. The absorbance of THP-1 macrophages without TG treatment was set as $100 \%$. (G) THP-1 macrophages were incubated with TG in the presence of glyburide for $24 \mathrm{~h}$ and caspase-2 activity was assessed. All data are expressed as the mean \pm SEM of three independent experiments. P-values were determined with Student's t-test. $* \mathrm{P}<0.05, * * \mathrm{P}<0.01, * * * \mathrm{P}<0.001$. 
ATP is involved in TG-induced apoptotic cell death, PMAdifferentiated THP-1 macrophages were incubated with TG in the presence or absence of ATP to assess cell viability. Treatment of THP-1 macrophages with ATP and TG resulted in a greater reduction in cell viability compared to treatment with TG only (Fig. 2A). Subsequently, to investigate whether extracellular ATP enhances apoptotic cell death in TG-treated macrophages, PARP cleavage was confirmed by western blot analysis. Extracellular ATP increased the levels of cleaved PARP in TG-treated macrophages (Fig. 2B). In addition, extracellular ATP also increased the activity of caspase- 1 and 2 in TGstimulated macrophages (Fig. 2C and 2D). To elucidate whether the increase in extracellular ATP was responsible for an increased TG-triggered apoptotic cell death, THP-1 macrophages were treated with TG in the presence of the ATP-depleting enzyme apyrase, which catalyzes the hydrolysis of ATP and ADP to AMP and inorganic phosphate. Cell viability was recovered (Fig. 2E) and the cleavage of PARP and apoptotic caspases (caspase-3, -7, -8, and -9) was reduced in the presence of apyrase in TG-treated THP-1 macrophages (Fig. 2F).
Moreover, ATP depletion decreased the activity of caspase-1 and 2, which was increased in response to TG treatment (Fig. $2 \mathrm{G}$ and $2 \mathrm{H}$ ). These results suggested that extracellular ATP is associated with TG-triggered macrophage death.

\section{Activation of the pannexin-1 channel mediates TG-induced THP-1 macrophage cell death}

Pannexin-1 channels are mechanosensitive conduits for ATP release in human cells (17). These channels are activated upon the cleavage of their C-terminus, and as they are activated, their cleavage fragments accumulate in the intracellular pool (18). To elucidate whether pannexin-1 is involved in macrophage cell death following TG treatment, THP-1 macrophages were treated with TG, and cytoplasmic pannexin-1 cleavage was measured using Western blot analysis. As a result, TG treatment increased cleaved pannexin-1 in a dose-dependent manner and pannexin-1 cleavage began to increase from $1.5 \mathrm{~h}$ after treatment (Fig. 3A).

When the pannexin- 1 channel is activated, both ATP and small dye molecules can pass through it (18). An Et-Br uptake
A

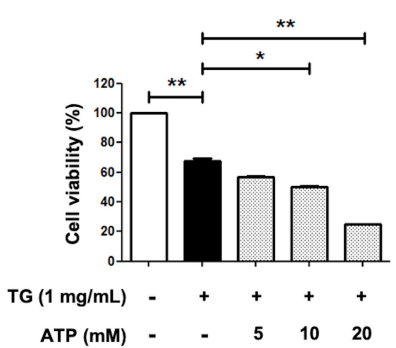

E

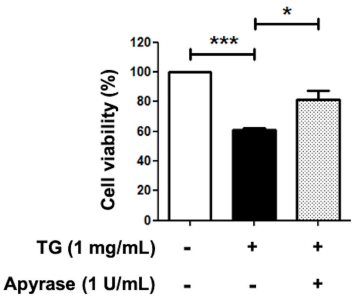

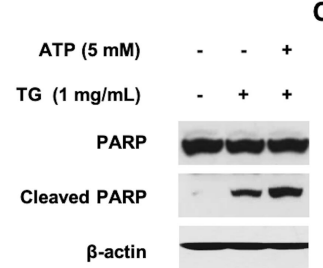

$\mathbf{F}$

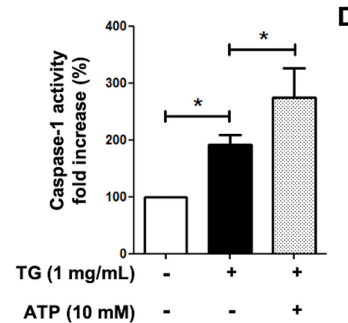

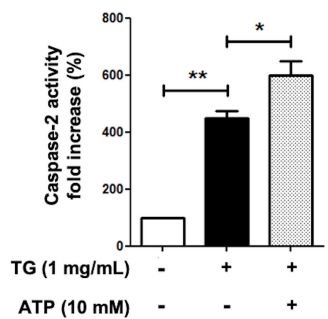

H

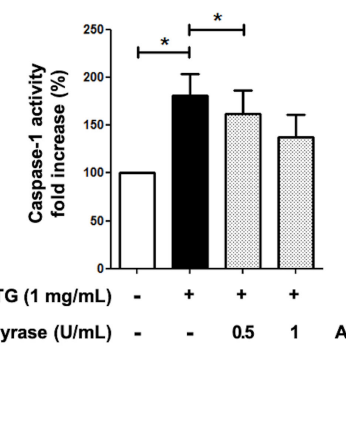

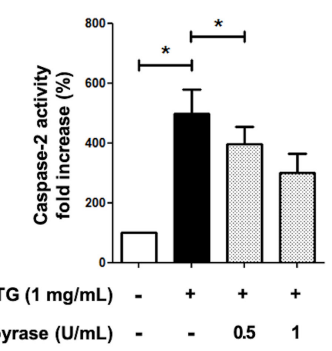

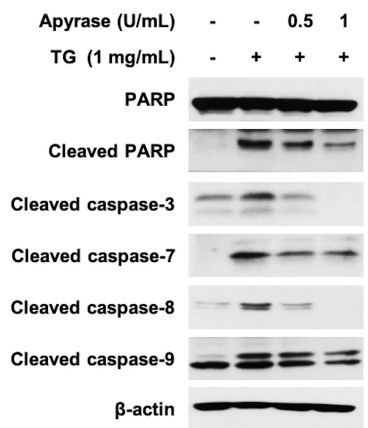

Fig. 2. An increase in extracellular ATP induces TG-triggered macrophage cell death. (A) THP-1 macrophages were incubated with TG in the presence of ATP for $24 \mathrm{~h}$ and the trypan blue exclusion assay was performed. The number of viable cells in THP- 1 macrophages without TG treatment was set as $100 \%$. (B) The cleaved form of PARP was detected by Western blotting. (C) THP-1 macrophages were incubated with TG in the presence of ATP for $24 \mathrm{~h}$, after which caspase-1 activity was assessed. The absorbance of THP-1 macrophages without treatment TG was set as 100\%. (D) THP-1 macrophages were incubated with TG in the presence of ATP for $24 \mathrm{~h}$ and caspase-2 activity was assessed. (E) THP-1 macrophages were incubated with TG in the presence of the ATP hydrolysis enzyme apyrase for $24 \mathrm{~h}$, and viable cells were enumerated. The number of viable cells in THP-1 macrophages without TG treatment was set as $100 \%$. (F) The cleaved form of PARP, and the cleavage of caspase- $3,-7,-8$, and -9 were detected by Western blotting. (G) THP-1 macrophages were incubated with TG in the presence of apyrase for $24 \mathrm{~h}$, after which caspase-1 activity was assessed. The absorbance of THP-1 macrophages without TG treatment was set as $100 \%$. (H) THP-1 macrophages were incubated with TG in the presence of apyrase for $24 \mathrm{~h}$ and caspase-2 activity was assessed. All data are expressed as the mean \pm SEM of three independent experiments. P-values were determined with Student's t-test. ${ }^{*} \mathrm{P}<0.05,{ }^{* *} \mathrm{P}<0.01, * * * \mathrm{P}<0.001$. 
A

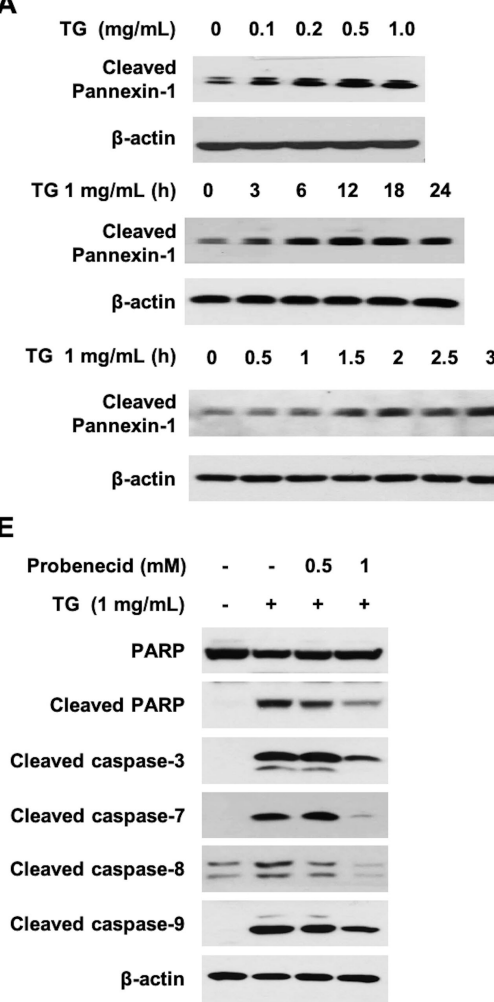

B

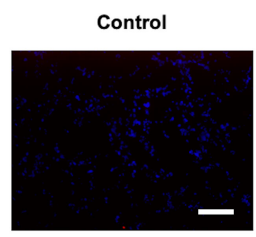

C
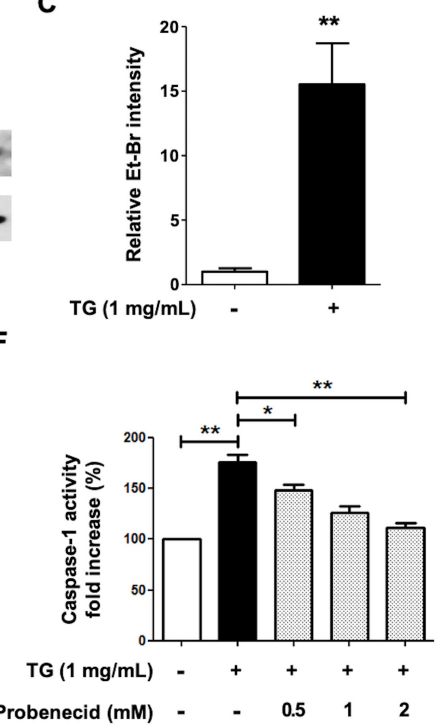

D
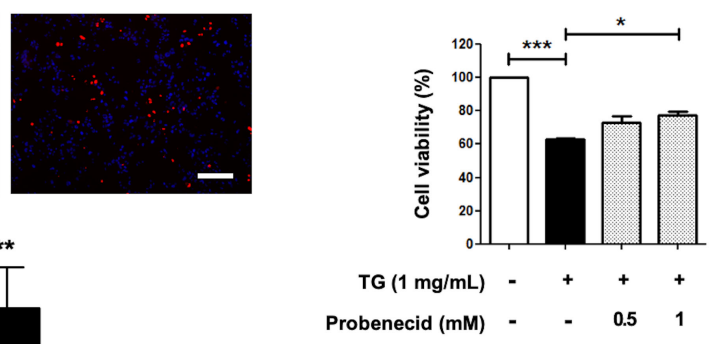

G

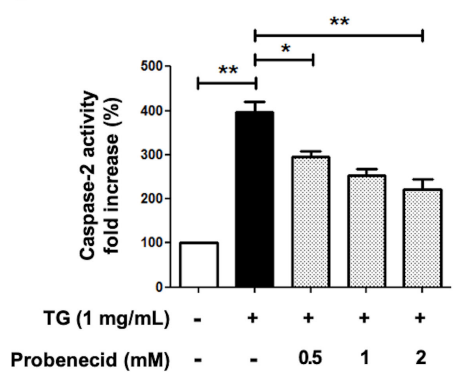

Fig. 3. TG-induced cell death involves activation of pannexin-1 channel in THP-1 macrophages. (A) THP-1 macrophages were incubated with the indicated concentration of TG for the indicated times. The cleaved form of pannexin-1 was detected by Western blotting. (B) THP-1 macrophages were incubated with or without TG for $24 \mathrm{~h}$ and an ethidium bromide uptake assay was performed to detect dye uptake by pannexin-1 channels (scale bars, $200 \mu \mathrm{m}$ ). (C) Relative ethidium bromide intensity measured. The fluorescence intensity of THP-1 macrophages without TG treatment was set as 1.0. (D) THP-1 macrophages were incubated with TG in the presence of the pannexin-1 channel inhibitor probenecid for $24 \mathrm{~h}$, then viable cells were enumerated. The number of viable cells in THP-1 macrophages without TG treatment was set as $100 \%$. (E) The cleaved form of PARP, and the cleavage of caspase-3, $-7,-8$, and -9 were detected by Western blotting. (F) THP-1 macrophages were incubated with TG in the presence of probenecid for $24 \mathrm{~h}$, after which caspase-1 activity was assessed. The absorbance of THP-1 macrophages without TG treatment was set as 100\%. (G) THP-1 macrophages were incubated with TG in the presence of probenecid for $24 \mathrm{~h}$ and caspase-2 activity was assessed. All data are expressed as the mean \pm SEM of three independent experiments. P-values were determined with Student's t-test. ${ }^{*} \mathrm{P}<0.05, * * \mathrm{P}<0.01, * * * \mathrm{P}<0.001$.

assay was performed to evaluate the activation of the pannexin-1 channel after THP-1 macrophages were treated with TG. More fluorescent red dots were observed in TG-treated macrophages than those that were not (Fig. 3B and 3C). These results show that TG treatment increases pannexin- 1 channel activity in THP-1 macrophages.

To determine whether the activity of pannexin-1 is involved in TG-induced macrophage death, TG-treated THP-1 macrophages were treated with probenecid, an inhibitor of pannexin-1. When the activity of pannexin-1 in TG-treated THP-1 macrophages was inhibited by probenecid, cell viability was recovered (Fig. 3D), and the cleavage of caspase- $3,-7,-8$, and -9 , and PARP were decreased (Fig. 3E). The activity of caspase- 1 and caspase-2 in TG-treated THP-1 macrophages was also reduced (Fig. 3F and 3G). Taken together, these results indicate that pannexin-1 channel activation mediates TG-triggered cell death in THP-1 macrophages.

\section{TG-triggered cell death is mediated by ATP released by} pannexin-1 channel activation

To test whether the activation of the pannexin- 1 channel contributes to extracellular ATP-dependent caspase- 1 and -2 activation and increases TG-induced cell death, THP-1 macrophages were incubated with TG in the presence of probenecid and ATP. The cell viability recovered upon the inhibition of the pannexin-1 channels was reduced again by the presence of the extracellular ATP in a dose-dependent manner (Fig. 4A). In addition, the cleavage of the caspases and PARP was increased again in response to the presence of ATP (Fig. 4B). The activity of caspase-1 and 2 decreased upon probenecid treatment was increased again in response to ATP treatment in a dose-dependent manner (Fig. 4C and 4D). Taken together, these results suggest that the 
A

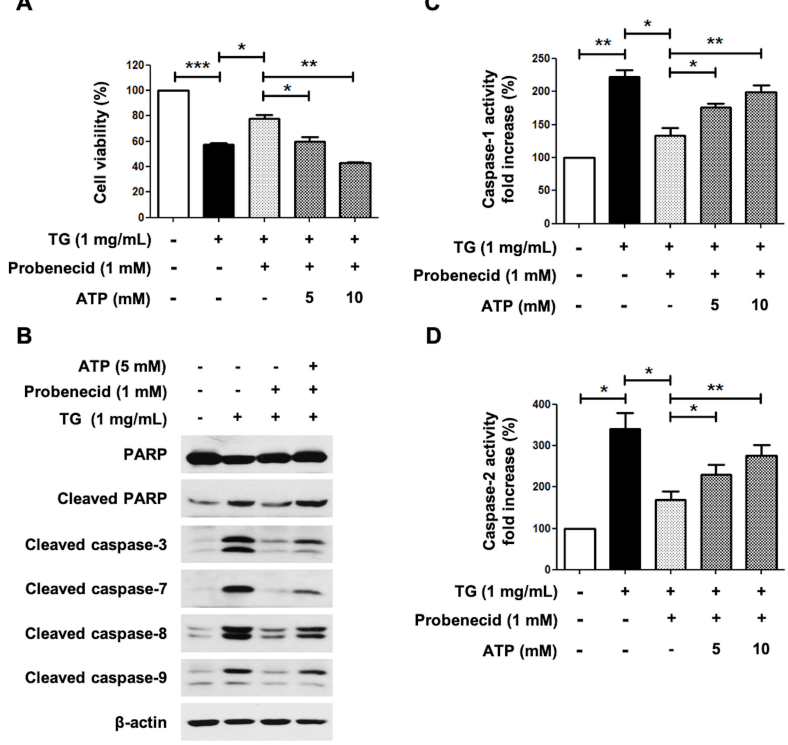

Fig. 4. TG-triggered cell death is mediated by ATP released via activated pannexin-1 channels. (A) THP-1 macrophages were incubated with TG in the presence of probenecid and ATP for $24 \mathrm{~h}$ and viable cells were enumerated. The number of viable cells in THP-1 macrophages without TG treatment was set as $100 \%$. (B) The cleaved form of PARP, and the cleavage of caspase- $3,-7,-8$, and -9 were detected by Western blotting. (C) THP-1 macrophages were incubated with TG in the presence of probenecid and ATP for $24 \mathrm{~h}$, after which caspase-1 activity was assessed. The absorbance of THP-1 macrophages without TG treatment was set as $100 \%$. (D) THP-1 macrophages were incubated with TG in the presence of probenecid and ATP for $24 \mathrm{~h}$ then caspase-2 activity was assessed. All data are expressed as the mean \pm SEM of three independent experiments. P-values were determined with Student's t-test. $* P<0.05, * * P<0.01, * * * P<0.001$.

activation of the pannexin-1 channel mediates ATP-dependent TG-triggered cell death in THP-1 macrophages.

\section{DISCUSSION}

Macrophage cell death is an essential phenomenon in atherosclerosis, contributing to the development of plaques in advanced lesions (19). Since Oxi-LDL has been considered a key player in the development of atherosclerosis, much research has been directed at understanding the association between Oxi-LDL and macrophage death $(20,21)$. In contrast to Oxi-LDL, the role of TG in atherosclerosis has long been controversial. However, emerging evidence, including a recent large cohort study, suggested that elevated TG levels can be a causal risk factor for atherosclerosis (22). Thus, in the current study, we showed that i) TG increased extracellular ATP via pannexin-1, which in turn, ii) activates ATP-sensitive potassium channels, and that iii) there is an activation of the caspase cascade through increased potassium efflux. These results suggest that the TG-induced cell death of macrophages is mediated by pannexin-1 activation.

Pannexin-1 was identified as a plasma membrane hemichannel that releases ATP from the cytosol to the extracellular space during apoptosis (23). The released ATP can act as a "find-me" signal to recruit phagocytes and activate the initiation of inflammation, which can be an important contributor to the pathogenesis of atherosclerosis (23). We previously demonstrated that TG treatment enhanced the secretion of a proinflammatory cytokine, IL-1 $\beta$, in THP-1 macrophages (6). Another function of increased extracellular ATP is the activation of ATP-sensitive potassium channels, subsequently leading to an increase in potassium efflux. Several investigations suggested that potassium channel activation contributes to the pathogenesis of atherosclerosis $(24,25)$. Ling and colleagues showed that the inhibition of ATP-sensitive potassium channels ameliorated atherosclerosis in $\mathrm{ApoE}^{-/-}$mouse models, directly elucidating the role of ATP-sensitive potassium channels in atherosclerosis (25). Moreover, they observed that the number of ATP-sensitive potassium channels in macrophages increased in atherosclerotic plaques, which may be explained by the present study. Here, we showed that TG-mediated macrophage cell death was dependent on the activation of ATP-sensitive potassium channels. It is possible that increased ATP-sensitive potassium channels in macrophages cause an increase in potassium efflux, leading to macrophage cell death. This may then potentiate the development of more vulnerable plaques.

In conclusion, our results provide a novel mechanism regarding how TG induces macrophage cell death. Since an enhanced level of macrophage death is considered a crucial phenomenon in plaque development in atherosclerosis, our results imply that TG-induced macrophages can create plaques and produce thrombi, resulting in clinical ischemic cardiomyopathy. Furthermore, our results support that TG can be a risk factor in the pathogenesis of atherosclerosis, and that control of TG levels can be a valuable therapeutic target for the disease.

\section{MATERIALS AND METHODS}

See supplementary information for Materials and Methods.

\section{ACKNOWLEDGEMENTS}

This research was supported by the Korea Nazarene University Research Grant 2020.

\section{CONFLICTS OF INTEREST}

The authors have no conflicting interests.

\section{REFERENCES}

1. Lozano R, Naghavi M, Foreman K et al (2012) Global and regional mortality from 235 causes of death for 20 age groups in 1990 and 2010: a systematic analysis for the 
Global Burden of Disease Study 2010. Lancet 380, 2095-2128

2. Rafieian-Kopaei M, Setorki M, Doudi M, Baradaran A and Nasri H (2014) Atherosclerosis: process, indicators, risk factors and new hopes. Int J Prev Med 5, 927-946

3. Parthasarathy S, Raghavamenon A, Garelnabi $M O$ and Santanam N (2010) Oxidized low-density lipoprotein. Methods Mol Biol 610, 403-417

4. Thomsen M, Varbo A, Tybjaerg-Hansen A and Nordestgaard BG (2014) Low nonfasting triglycerides and reduced allcause mortality: a mendelian randomization study. Clin Chem 60, 737-746

5. Bersot T, Haffner S, Harris WS, Kellick KA and Morris CM (2006) Hypertriglyceridemia: management of atherogenic dyslipidemia. J Fam Pract 55, S1-8

6. Son SJ, Rhee K-J, Lim J, Kim TU, Kim T-J and Kim YS (2013) Triglyceride-induced macrophage cell death is triggered by caspase-1. Bio Pharm Bull 36, 108-113

7. Lim J, Kim HK, Kim SH, Rhee KJ and Kim YS (2017) Caspase-2 mediates triglyceride (TG)-induced macrophage cell death. BMB Rep 50, 510-515

8. Park IS and Kim JE (2002) Potassium efflux during apoptosis. J Biochem Mol Biol 35, 41-46

9. Abdalah R, Wei L, Francis K and Yu SP (2006) Valinomycininduced apoptosis in Chinese hamster ovary cells. Neurosci Lett 405, 68-73

10. Imre G, Heering J, Takeda AN et al (2012) Caspase-2 is an initiator caspase responsible for pore-forming toxin-mediated apoptosis. EMBO J 31, 2615-2628

11. Tinker A, Aziz Q and Thomas A (2014) The role of ATPsensitive potassium channels in cellular function and protection in the cardiovascular system. Br J Pharmacol $171,12-23$

12. Coetzee WA (1992) ATP-sensitive potassium channels and myocardial ischemia: why do they open? Cardiovasc Drugs Ther 6, 201-208

13. Ruan Z, Orozco IJ, Du J and Lu W (2020) Structures of human pannexin 1 reveal ion pathways and mechanism of gating. Nature 584, 646-651

14. Bortner CD, Hughes FM Jr and Cidlowski JA (1997) A primary role for $\mathrm{K}+$ and $\mathrm{Na}+$ efflux in the activation of apoptosis. J Biol Chem 272, 32436-32442

15. Yu SP, Yeh CH, Gottron F, Wang X, Grabb MC and Choi DW (1999) Role of the outward delayed rectifier K+ current in ceramide-induced caspase activation and apoptosis in cultured cortical neurons. J Neurochem 73, 933-941

16. Brough D, Pelegrin P and Rothwell NJ (2009) Pannexin-1dependent caspase-1 activation and secretion of IL-1beta is regulated by zinc. Eur J Immunol 39, 352-358

17. Bao L, Locovei S and Dahl G (2004) Pannexin membrane channels are mechanosensitive conduits for ATP. FEBS Lett 572, 65-68

18. Penuela S, Gehi R and Laird DW (2013) The biochemistry and function of pannexin channels. Biochim Biophys Acta $1828,15-22$

19. Tabas I (2009) Macrophage apoptosis in atherosclerosis: consequences on plaque progression and the role of endoplasmic reticulum stress. Antioxid Redox Signal 11, 2333-2339

20. Salvayre R, Auge N, Benoist H and Negre-Salvayre A (2002) Oxidized low-density lipoprotein-induced apoptosis. Biochim Biophys Acta 1585, 213-221

21. Giovannini C, Vari R, Scazzocchio B et al (2011) OxLDL induced p53-dependent apoptosis by activating p38MAPK and PKCdelta signaling pathways in J774A.1 macrophage cells. J Mol Cell Biol 3, 316-318

22. Klempfner R, Erez A, Sagit B-Z et al (2016) Elevated triglyceride level is independently associated with increased all-cause mortality in patients with established coronary heart disease: twenty-two-year follow-up of the bezafibrate infarction prevention study and registry. Circ Cardiovasc Qual Outcomes 9, 100-108

23. Chekeni FB, Elliott MR, Sandilos JK et al (2010) Pannexin 1 channels mediate 'find-me'signal release and membrane permeability during apoptosis. Nature 467, 863-867

24. Schilling T and Eder C (2009) Lysophosphatidylcholineand MCP-1-induced chemotaxis of monocytes requires potassium channel activity. Pflugers Arch 459, 71-77

25. Ling MY, Ma ZY, Wang YY et al (2013) Up-regulated ATP-sensitive potassium channels play a role in increased inflammation and plaque vulnerability in macrophages. Atherosclerosis 226, 348-355 